\title{
Different Kinds of Near-Death Experience: A Report on a Survey of Near-Death Experiences in Germany
}

\author{
Prof. Dr. Hubert Knoblauch \\ University of Zürich, Switzerland \\ Ina Schmied, M.A. \\ Institut für Grenzgebiete der Psychologie und \\ Psychohygiene, Freiburg i. Br., Germany \\ Bernt Schnettler, M.A. \\ University of Konstanz, Germany
}

\begin{abstract}
This article provides a short summary of a representative survey on near-death experiences (NDEs) in Germany, which is the first of its kind in Europe. We tested several assumptions derived from previous research on NDEs, including the assumptions of a unified pattern of experience, the universality of the pattern, and the necessary link between NDEs and clinical death. We received replies from more than 2,000 persons, 4 percent of whom reported NDEs. The patterns of the NDEs did not seem to correspond to earlier findings: aside from being much more diverse, they also differed with respect to cultural variables, particularly the difference between religious interpretations and the differences between post-socialist East Germany and West Germany.
\end{abstract}

KEY WORDS: sociology of near-death experience; surveys; Europe; Germany; cultural comparison.

Dr. Hubert Knoblauch is currently professor of Religious Studies at the University of Zürich, Switzerland. Ina Schmied is researcher at the IGPP at Freiburg, Germany, and Bernt Schnettler is researcher at the University of Konstanz, Germany. The research project described in the paper was conducted by Hans-Georg Soeffner, Hubert Knoblauch, Ina Schmied and Bernt Schnettler, and was supported by the Institut für Grenzgebiete der Psychologie und Psychohygiene (IGPP), Freiburg, Germany. The results of the study, of which we can present here only small parts, are published in: H. Knoblauch, H.G. Soeffner (eds.), Todesnaehe, Konstanz: UVK 1999. Requests should be addressed to Prof. Dr. Hubert Knoblauch at the University of Zürich, Religious Studies, Kirchgasse 9, CH-8001 Zürich, Switzerland; e-mail: Hubert.Knoblauch@access.unizh.ch. 
Reports on near-death experiences have become almost a cultural fashion. Television shows, magazines, newspaper, and other media frequently present people who talk about experiences of this kind. Nevertheless, we know little as to how many people have had experiences of this kind, and we do not know the range of experiences involved. Our research project at the University of Konstanz, Germany, on structure and distribution of near-death experiences tried to address these questions. The project aimed at a representative survey of near-death experiences (NDEs) in the Federal Republic of Germany and an analysis of the structure of the corresponding reports of NDEs.

Near-death experiences are reported by people who lived through a situation in which they were felt to be near death, dying, or even dead. Typically these experiences are considered as different from everyday life, and they involve different elements, stages, or motifs. Near-death experiences are sometimes treated as authentic reports of the transcendent reality lying beyond the normal realms of life; or as constructions of consciousness within a body in crisis. Some more critical observers of these phenomena regard them as forms of stories similar to the genre of urban legends, that is, as products of imagination.

The phenomenon covered by the notion of the NDE has been common throughout history. Reports of such experiences are known from pre-Christian history, ranging from Sumerian to classical Greek culture (Moraldi, 1987), and they are also mentioned in the Bible (2 Corinthians 12:1-4). As Peter Dinzelbacher (1985) found, reports on NDEs as subjective experiences of death constitute one of the most popular genres in the Middle Ages. At that time, the genre tended to become fictionalized in literature, adapting elements from mysticism and Christian iconology (Dinzelbacher, 1991). In Protestantism, the early 18th century saw a mounting number of reports about experiences near death, which typically functioned as moral instructions (Clark, 1852). In the 19th century, death books played an important role, containing statements by dying persons and regarded as warrants for the claim that dying did not necessarily mean the end of existence (Zaleski, 1995).

Scientific investigations of NDEs started at the end of the 19th century. The first systematic collection of reports of NDEs was assembled by the Swiss geologist Albert Heim (1892), who interpreted reports by mountain climbers who had survived serious falls. He found these reports to be strongly religious in content and showed that 95 percent of such accident victims experienced being near death as exceedingly pleasant. In 1926, the British physicist William Barrett published a study on deathbed visions that included near-death experiences. Yet, 
although the phenomenon had been discussed before (Scott, 1931; Tucker, 1943), it was only in more recent times that near-death experiences triggered what may be called a cultural fashion. One major influence on this development has been the popular books of psychiatrist Raymond Moody (1975), who interviewed 150 people and found recurring elements in their reports, which he combined into what he constructed as the ideal case. Typically, this ideal case was described in terms of 15 consecutive elements, such as the notice of one's own death, followed by a feeling of silence and inner peace, then by sounds. After hearing sounds one enters something that resembles a dark tunnel, then seems to be leaving one's body. Descriptions also include meeting others, perception of light or a creature of light, and a panorama of one's life.

\section{Assumptions About NDEs}

Following Moody, other researchers studied this phenomenon, including Stanislav Grof and Joan Halifax (1977), Russell Noyes and Roy Kletti (1977), Kenneth Ring (1980), and Michael Grosso (1981). In much of this research, a number of assumption became accepted, which we subjected to further scrutiny.

\section{Link to Biological Death}

The first such assumption is that NDEs are linked to the biological death. This assumption holds that NDEs occur only to subjects who have been medically in the most dangerous conditions, such as clinical death. This assumption led several investigations to concentrate solely on people who have been near death. Thus Fred Schoonmaker (Audette, 1979) restricted his studies to survivors of medically lifethreatening situations, as did Karlis Osis and Erlendur Haraldsson (1977) and Michael Sabom (1982).

\section{Common Structure or Schema}

Many researchers assume that NDEs exhibit a common structure and manifest a unified, common schema (Zaleski, 1995). Despite the commonality of this assumption, investigators often disagree as to what elements form part of the basic structures. Whereas Moody mentioned 15 elements, Michael Schröter-Kunhardt (1993) found 11; Sabom (1982) identified ten elements, as did George Gallup and William Proctor 
(1982); and Ring (1980) found NDEs to be comprised of five phases. In general, one can identify two lines of research.

Common Motifs and Temporal Sequences. First, in the tradition of Moody, many prominent researchers have maintained that the structure of NDEs is constituted predominantly by certain substantial elements, or motifs, by which we mean the content of what is experienced or its noemic qualities, such as a tunnel, a light, and an out-of-body experience. Like Moody, these researchers also have assumed that these motifs occur one after another, that is, that the NDE exhibits a certain sequentiality, which is often considered to be very clear-cut. Thus Ring (1980) identified five different stages of inner peace, the out-of-body experience, entering a darkness, perception of light, and entering the light, which he assumed succeed one another. We should note, in discussing these elements or motifs, that some of them have also been studied in isolation, such as out-of-body experiences, tunnels, and panoramic experiences (Blackmore, 1982; Drab, 1981; Noyes and Kletti, 1977).

Common Forms of Experience. The other line of research assumes that NDEs may be described in terms of forms of experiences, by which we mean the ways in which the experience os constructed, or its noetic quality. In this vein, Sabom (1982) tended to use more abstract categories related to the study of religious experience. To him, NDEs are characterized by ineffability, timelessness, a sense of reality, a feeling of being dead, acoustical perceptions, bodily separation, and a mental journey and return. Bruce Greyson (1985) characterized NDEs by four core dimensions-cognitive, affective, paranormal, and transcendentalwhich we have included in our questionnaire.

Other Structural Assumptions. Aside from the typical motifs of NDEs, there are some additional elements that are often taken for granted as being part of a common structure of NDEs. These include four commonly accepted hypotheses.

The first hypothesis is that NDEs lead to fundamental changes in life. On the one hand, they may affect only the subject's image of and attitude to death; on the other hand, they may lead to serious changes and aftereffects on the subject's life style, ranging from changes in social behavior to increased religiousness. According to P. M. H. Atwater (1988), Charles Flynn (1986), Ring (1992), Cherie Sutherland (1990, 1995/1992), and others, NDErs tend towards postmaterialist values; some believe that they have been chosen for some unique but still unknown mission, and that God or some other supernatural force was 
responsible for their new sense of destiny. Many have a new or intensified belief in some form of life after death, including an increased belief in reincarnation (Wells, 1993).

The second hypothesis is that NDEs are ineffable, that is, they are said to be expressed in language and communication only with great difficulty and loss of content. The third hypothesis is that NDEs are a taboo subject in most communicative contexts. Experiencers typically hide their NDEs and communicate them only under special circumstances. And the fourth hypothesis is that NDEs are of a distinctly positive nature. Although there are a few indications that some NDEs are experienced as unpleasant, bad, or "hellish," a common assumption is still that NDEs are of a distinctly positive nature.

\section{Universality}

A third major assumption is that NDEs follow "universal laws of symbolic experience" (McClenon, 1994, p. 172) that remain constant across cultural as well as social differences. Thus Glenn Roberts and John Owens (1988) wrote that "The central features of the NDE have been recorded throughout history and across numerous cultures and religious groups" (p. 611). And Schröter-Kunhardt (1990) concluded that NDEs exhibit outstanding cross-cultural similarities of form, despite considerable diversity of imagery and content, that is, recurrent motifs, composite imagery, and a series of sequential events. We shall refer to this assumption as the universality assumption. In a more sophisticated version the assumption concedes that there are differences in the structure of experience; yet, these differences are said to be accounted for by different degrees of elaboration and depth of investigation of other accidental circumstances.

\section{Religious Interpretations}

The fourth assumption is that NDEs are linked to religious interpretations in two different ways. On the one hand, the assumption of a universal structure of the NDE led some investigators to speculate about a religious explanation for these experiences, by postulating either the objective existence of the reality experienced (Kübler-Ross, 1969) or the existence of a religious faculty of human experience, which supposedly forms the basis of the universal structure (Roberts and Owen, 1988).

Secondly, and on a more empirical basis, some investigators found that these experiences are regarded as religious by the experiencers. 
Thus Ring (1982) wrote that "A specifically religious interpretation is given to it by many, though not all, of the core experiencers" (p. 138). The same was observed by James McClenon (1993) in his interviews of American and Japanese students. Moreover, the NDE is said to increase individual religiosity (Schröter-Kunhardt, 1990).

\section{Study Rationale and Methods}

In general, we started from the assumption that one must distinguish between experiences of near death and reports of NDEs. As scientific observers, we have access only to reports of NDEs. This also holds for those studies that claim to investigate experiences. Actually, reports on NDEs are, in general communicatively constituted accounts of what is claimed to have been experienced subjectively. Parenthetically, there is an interesting issue on the relation between experience, communication, and culture, which, however, cannot be pursued at this point. Suffice it to say that our sociological interests are strongly related to this issue. On the grounds of this assumption we infer that there must be social and cultural influences on NDE reports, since the communication of experiences is necessarily a social activity. Our main thesis, therefore, was that the structure of reports of NDEs varies, and that it is subject to cultural and social factors.

The overall goal of the research project was to pursue two questions: (1) How many people have had these experiences? and (2) What are the structures of these experiences? Both questions were analyzed with respect to their relation to social and cultural variables. Of particular interest to us was a comparison of East German and West German data, since those two societies differ with respect to some crucial cultural features.

Since the distribution of NDEs was one of our two main questions, we had to survey a sample representing the whole society. In order to achieve this goal, we collaborated with a recognized institute specializing in surveys, ZUMA at Mannheim, which conducted the survey.

The survey was preceded by a series of 18 qualitative interviews conducted by Hubert Knoblauch. In addition to available investigations of similar kinds, we drew on the results of these interviews in the development of the questionnaire. The entire survey sample were asked if they had ever had an NDE or similar experience. Following this filtering question, the rest of the survey was administered only to those who gave positive or ambivalent answers (for example, "I am not sure") 
to this first question. Survey respondents were asked to fill out the questionnaire by themselves. In addition to these data, we also had access to the sociodemographic data of our sample and of the general survey.

The questionnaire consisted of a series of questions, including multiple-choice scales, as well as open-ended questions that had been tested in advance. In order to access the structure of the experience, we formulated a multiple-choice question including several different items, motifs, and features mentioned by earlier researchers. In addition, we asked respondents to describe the content of their experience in an open-ended question. The questionnaire also included questions about the circumstances of the experience, its interpretation, and its religious meaning. The structured questions were analyzed statistically, using loglinear models to explain the influences on NDEs. We also applied cluster analysis for the classification of particular experiences. Open-ended questions, on the other hand, were interpreted by qualitative methods which considered (a) the form of recounting, or narrative structure, and (b) topical contents, or motifs.

\section{Results and Discussion}

\section{Frequency of Experiences}

Of the 2,044 persons interviewed, 356 answered positively to having had experiences with death of at least one of the following kinds (some respondents reported more than one kind of experience): 258 respondents (14 percent) reported a premonition of someone else's death that, in their view, later proved true; 79 respondents (4 percent) reported having witnessed deathbed visions; and 55 respondents ( 2.8 percent) reported having seen paranormal phenomena while being with dying persons, such as a cup breaking at the moment of death.

With respect to the NDE, 118 persons (6 percent) completed the entire questionnaire. However, that number exaggerates the percentage of NDErs in the sample, since it included respondents who stated that they were not sure whether they had had an NDE. On the basis of the multiple-choice and open-ended questions, we eliminated those questionnaires that described experiences not directly related to NDEs, such as death shocks, death presentiments, and reports of NDEs occurring to persons other than the respondent. With those extraneous questionnaires eliminated, we found that 82 respondents (4 percent of the sample) reported an NDE. 
It should be stressed that this can be considered the first representative survey of the frequency of NDEs in Germany. Since our sample was representative of the German population in a statistical sense, with respect to the total population of 82 million in the 1995 German census, the results suggest that about 3 million Germans have had such an experience. The sample exhibited a very symmetrical distribution between East (40 NDErs) and West Germany ( 42 NDErs); and between men (41 NDErs) and women (41 NDErs), again with a symmetric distribution in East (20 men and 20 women) and West Germany (21 men and 21 women). The average age of the experiencers was 36 years. NDEs dated back from 1 to 65 years, the average being 13 years. The percentage of NDEs with respect to the gender of the sample was slightly higher for men (4.3 percent) than for women (3.8 percent).

Whereas our data on the distribution of the NDE can be taken as statistically representative, our findings with respect to other variables can be taken to indicate only tendencies. Nevertheless, some of these tendencies do allow us to address quite clearly the assumptions mentioned above.

\section{Link to Biological Death}

As to the question of whether NDEs are typically linked to biological death, our data gave a very clear indication, with survey questions concerning both subjective and objective evidence of biological death. In fact, less than 50 percent of the respondents claimed to have been in a life-threatening situation when experiencing an NDE, and only 6 percent claimed to have been clinically dead.

\section{Structure and Universality of NDEs}

The fact that the questionnaire allowed for open-ended questions yielded particularly interesting results with respect to the structure of the NDE. By analyzing the descriptions of NDEs in close detail, we found some recurrent patterns, which suggested different types of NDE. Surprisingly, the most detailed and explicit description of NDEs did not correspond to those reported by Moody, Ring, and others. Instead of consisting of a sequence of different events characterized by abstract motifs, they consisted of singular scenes, the content of which varied immensely. For example, one person near death reported a scene in which a figure with a scythe tried to grasp her hand, and another scene in which he accompanied her to the door and was pushed out the door. 
Some of these scenes were experienced in some detail, yet they hardly exhibited any sequence of events or motifs.

The less elaborate descriptions were devoted to single motifs. However, even in these cases there were none that corresponded, for example, to Ring's stages. One might argue that the focus on single motifs might have been overcome if one conducted in-depth interviews. Yet, as mentioned above, we did conducted a series of in-depth interviews, and their results tended to support the findings in our survey. The structure of the experiences could be delineated according to different types. Although it must be admitted that a number of cases lay between the types, the majority of the written accounts fell into on of the following categories.

One type may be labeled "being in transcendence." Reports of this kind were characterized by claims that experiences occurred in a transcendent reality that differed from the everyday life or world. Although this reality was compared to heaven or paradise, other beings were mentioned rarely, and the descriptions surprisingly lacked references to God or godlike entities. Another type of report focused on the emotional quality of the experience, ranging from "indifference" to "great feeling." Other beings or activities were rarely mentioned. In terms of the specific emotions experienced, 50 percent of the NDErs reported positive emotions, and 43 percent negative emotions.

A range of reports highlighted the contrast between light and dark, which was understood in visual terms. These experiences lacked emotional features and the respondents took an almost distanced observational stance. In only a few cases this type overlapped with reports of experiences that may be labeled as out-of-body experiences. And wherever we found descriptions of panoramic memory or life review experiences, they were not linked to any other feature.

One of the most frequent types could be called a "scenic experience." These consisted of often elaborate and detailed descriptions of single scenes, actions, or events that either appeared surreal, involved an encounter with other beings, or consisted of descriptions of landscapes comparable to the medieval descriptions of the locus amoenus.

Despite the different types of experiences, most of the respondents (63 percent) stressed that they felt very conscious while experiencing the NDE. Although a large majority of our respondents ( 80 percent) supported the ineffability hypothesis, that is, the view that NDEs can hardly be expressed in words, the assumption that NDEs are anathema in modern society did not fare as well. In fact, 73 percent of our respondents declared that they had met interested listeners when telling the 
story, 73 percent expected a strong interest in the topic by others, and almost 60 percent thought that their stories were believed.

Concerning the aftereffects of the NDE, the results of our sample contradicted previous findings. Although respondents supported general statements without objective behavioral consequences ( 70 percent reported an increased feeling of living more consciously, and 63 percent reported increased interest in the meaning of life), religious belief and belief in God increased for only 28 percent and remained the same for 67 percent. Surprisingly, fear of death decreased for only 40 percent, remained stable for 40 percent, and even increased for 20 percent!

The assumption of a common scheme of experiences across cultures was difficult to test. Nevertheless, some variables in our data related to this hypothesis. On the one hand, there seemed to be a certain relation between experiencing the NDE and knowledge about NDEs. Whereas 52 percent of the NDErs had known of the phenomenon, only 34 percent of the nonexperiencers had ever heard previously about NDEs. There were even greater differences between experiencers and nonexperiencers in the sources of their knowledge about NDEs, as indicated in Table I.

It is quite apparent that people have a lot of knowledge about NDEs, partly derived from mass media, whether or not they have had such an experience. The knowledge related either to the term NDE or to the definition of its content. It was also interesting that experiencers had more specialized knowledge than did nonexperiencers, for example, drawing on books or going to special events. The most striking indication in this table, however, is the importance of personal contacts. Our data suggest that the chance of experiencing a NDE is related to knowing someone else who either knows about NDEs or has experienced one.

\section{Table I}

\section{Percent of NDErs and NonNDErs Who Had Learned About NDEs from Various Sources}

\begin{tabular}{lcc}
\hline \multicolumn{1}{c}{ Source of Knowledge } & Experiencers & Nonexperiencers \\
\hline Near-death experiencers & 56 & 15 \\
Other persons & 56 & 20 \\
Radio and television & 74 & 82 \\
Newspapers and magazines & 77 & 72 \\
Books & 63 & 36 \\
Seminars and other events & 26 & 7 \\
\hline
\end{tabular}


There were also indications of cultural differences in our survey, based on the fact that Germany society had until recently been divided into two different ideological systems. It is quite sound to assume that, before the 1990s, East Germans had very little information and cultural communication about NDEs. On that assumption, we compared the results between East and West German respondents. First, it was surprising to learn that NDEs seem to have been experienced in the East as much as in the West. Ideological differences did not seem to affect the occurrence of NDEs. However, there were differences in the content of the experiences reported.

In general, NDEs were experienced much more positively in West Germany than in the East. Among West German NDErs, 60 percent reported positive emotions, and 29 percent negative; while among East German NDErs, only 40 percent reported positive emotions, and 60 percent negative. Also certain motifs were unequally distributed, such as tunnels being more common in the East, and light in the West. Percentages of experiencers who reported various features of NDEs are presented in Table II, categorized by geographical region, gender, and church membership. Statistical tests of the differences between these

Table II

Percent of NDErs Reporting Various NDE Motifs

\begin{tabular}{|c|c|c|c|c|c|c|c|}
\hline \multirow[b]{2}{*}{ Motif } & \multirow[b]{2}{*}{ Total } & \multicolumn{2}{|c|}{ Region } & \multicolumn{2}{|c|}{ Gender } & \multicolumn{2}{|c|}{$\begin{array}{c}\text { Church } \\
\text { Members }\end{array}$} \\
\hline & & West & East & Male & Female & Yes & No \\
\hline Full mental awareness & 65 & 67 & 63 & 61 & 68 & 65 & 63 \\
\hline Wonderful feelings & 50 & 60 & 40 & 44 & 56 & 46 & 57 \\
\hline Entering other realm & 48 & 55 & 40 & 44 & 51 & 48 & 47 \\
\hline Horrible feelings & 44 & 29 & 60 & 49 & 39 & 40 & 50 \\
\hline Life review & 44 & 43 & 45 & 49 & 39 & 44 & 43 \\
\hline Light & 40 & 50 & 30 & 32 & 49 & 40 & 40 \\
\hline Tunnel & 38 & 31 & 45 & 39 & 37 & 29 & 53 \\
\hline Heavenly realm & 38 & 45 & 30 & 29 & 46 & 37 & 40 \\
\hline Met living persons & 32 & 31 & 33 & 32 & 32 & 31 & 33 \\
\hline Out of body & 31 & 38 & 23 & 34 & 27 & 35 & 23 \\
\hline Feeling of being dead & 26 & 29 & 23 & 20 & 32 & 25 & 27 \\
\hline Contact with dead & 16 & 12 & 20 & 17 & 15 & 14 & 20 \\
\hline Horrible realm & 13 & 17 & 10 & 17 & 10 & 12 & 17 \\
\hline Met nonhuman beings & 11 & 12 & 10 & 19 & 12 & 12 & 10 \\
\hline
\end{tabular}


categories were not performed because of the small number of people in each group.

The influence of cultural factors was supported by the variable of religiosity. On the one hand, there were no striking differences between Roman Catholics, Protestants, and those with no religious affiliation, with the single exception that church members reported decidedly more NDEs than did those without church affiliation. The only exception was that respondents who identified themselves as members of a Christian sect other than Roman Catholic or Protestant had slightly more NDEs than other respondents. While this result suggests the influence of religious beliefs and socialization, it must be treated with caution, since the absolute numbers in this analysis are very low.

\section{Religious Interpretations}

There were more solid findings regarding religious interpretations of NDEs. Despite the fact that there are clear differences in religious affiliations between East Germany, where about 25 percent of the population are church members and atheism is predominant, and West Germany, where the majority of the population are still members of Roman Catholic or Protestant churches, Christian interpretations of the NDE were of little importance. Only 22 percent held a stronger believe in the Christian God, whereas 28 percent expected nothing to come after death. The most frequently endorsed interpretations were humanistic or diffuse transcendent beliefs, such as belief in a higher, supernatural power. These results differ from those in American surveys.

Table III

NDErs' Interpretations of Their Experiences

\begin{tabular}{lc}
\hline \multicolumn{1}{c}{ Interpretation } & Percent Agreeing \\
\hline Human life is valuable & 81 \\
There are transcendent entities & 59 \\
There are supernatural forces & 54 \\
No interpretation & 43 \\
There is a hereafter & 43 \\
There is a god & 35 \\
There is nothing after death & 28 \\
There is reincarnation & 27 \\
There is a Christian God & 22 \\
\hline
\end{tabular}


Whereas in America NDEs seem to support religious belief, in Germany they rather undermine it. NDEr's interpretations of their experiences are presented in Table III.

\section{Conclusions}

The results of the survey allow some quite clear answers to the problems posed. First, NDEs seem to be a widespread phenomenon in contemporary Germany, and a large part of the population knows about NDEs. Despite the common knowledge of NDEs, the experiences people reported differed quite substantially. One may, of course, argue that this finding depends on the subjective definition of an NDE. Yet all notions of near-death experience depend on subjective reports, so that one could argue that every reasonable definition of an NDE must be subjective.

In fact, the results indicate that a series of commonly held assumptions should be reconsidered. These include the assumption that NDEs are linked to biological death and the awareness of having been declared dead, the taboo against talking about the experience, and the consequences of the experience and its religious meaning. More critically, the results suggest that NDEs do not exhibit a common structure. Although following certain patterns, these patterns can hardly be subsumed under one structure. For this reason, the assumption that NDEs are a universal phenomenon also seems to be open to question.

As the data indicate, NDEs differ significantly not only between different experiencers according to several types; there are strong indications of cultural influences on NDEs. In this respect, the differences between East German and West German subjects suggest that sociocultural background affects not only the interpretation of NDEs but also the very content of what is experienced. That is, the content of NDEs is culturally constructed. Other researchers have provided further evidence for this assumption. Salteaux Indian NDEs contain different motifs, such as tipis (Hallowell, 1940); and there are clearly different interpretations among American and Marxist Chinese NDEs (Kellehear, 1996). In addition, Mormon NDEs differ in substantial ways from those described by Moody, Ring, and others (Lundahl, 1981-82).

This hypothesis seems also to hold with respect to religious affiliation. However, the most obvious cultural influence becomes salient if one compares the German data altogether with previously published studies, particularly on data from the United States, on which most of our literature review drew. Compared to American NDEs, the NDEs we found in Germany were significantly different in three aspects. 
The first significant difference was in the content in terms of motifs, sequences, and emotional quality of the experience. Whereas American NDEs include a certain number of elements that seem to follow one another and are typically experienced as emotionally positive, the majority of German NDEs consist of one element or scene without any temporal sequence of events or motifs. They are equally likely to be experienced either positively or negatively, although as noted above, there were salient differences between the samples from the two former German states. The second significant difference was in the consequences, in terms of effects of the experience on subsequent life. American NDEs affect experiencers' morality and lead to more or less serious changes in life. German NDEs, on the other hand, seem to affect only one's general outlook on life, without having moral consequences. The third significant difference was in the experiencers' interpretation with respect to worldview. Whereas American NDEs seem to strengthen religiosity and support existing religious beliefs, German NDEs are rarely considered religious at all.

Although these results indicate the importance of cultural influences, further research must examine these factors in more detail. Yet, despite the importance of cultural influences, we do not argue that NDEs are nothing but cultural constructions. To the contrary, we were rather surprised to find a large number of these experiences in the former German Democratic Republic, a society in which people had little access to knowledge about these experiences. One could, therefore, conclude that it is not the occurrence of the NDE that is influenced by culture, but its content and its interpretation.

\section{References}

Atwater, P. M. H. (1988). Coming back to life: The after-effects of the near-death experience. New York, NY: Dodd, Mead.

Audette, J. (1979). Denver Cardiologist discloses findings after 18 years of near-death research. Anabiosis: The Journal of Near-Death Studies, 1(1), 1-2.

Barrett, W. (1926). Death-bed visions: The psychical experiences of the dying. London, England: Methuen.

Blackmore, S. (1982). Beyond the body: An investigation of out-of-the-body experiences. London, England: Heinemann.

Clark, D. W. (1852). Death-bed scenes: Or dying with or without religion: Designed to illustrate the truth and power of Christianity. New York, NY: (publisher not identified).

Dinzelbacher, P. (1985). Mittelalterliche Visionen und moderne Sterbeforschung. In J. Kühnel (Ed.), Psychologie in der Mediävistik (pp. 9-49). Göppingen, Germany: Kümmerle.

Dinzelbacher, P. (1991). Revelationes. In Typologie de sources de moyen age occidental (p. 57). Turnhout: Brepols.

Drab, K. J. (1981). The tunnel experience: Reality or hallucination? Anabiosis: The Journal of Near-Death Studies, 1, 126-152. 
Flynn, C. F. (1986). After the beyond: Human transformation and the near-death experience. Englewood cliffs, NJ: Prentice-Hall.

Gallup, G., and Proctor, W. (1982). Adventures in immortality: A look beyond the threshold of death. New York, NY: McGraw-Hill.

Greyson, B. (1985). A typology of near-death experience. American Journal of Psychiatry, $142,967-969$.

Grof, S., and Halifax, J. (1977). The human encounter with death. New York, NY: Dutton.

Grosso, M. (1981). Toward an explanation of near-death phenomena. Journal of the American Society for Psychical Research, 75, 37-60.

Hallowell, I. (1940). The spirits of the dead in Salteaux life and thought. Journal of the Royal Anthropological Institute, 70, 29-51.

Heim, A. (1892). Notizen über den Tod durch Absturz. Jahrbuch des Schweizerischen Alpenclub, 27, 327-337.

Kellehear, A. (1996). Experiences near death: Beyond medicine and religion. New York, NY: Oxford University Press.

Kübler-Ross, E. (1969). On death and dying. New York, NY: Macmillan.

Lundahl, C. R. (1981-82). The perceived other world in Mormon near-death experiences. Omega, 12, 319-327.

McClenon, J. (1993). Surveys of anomalous experience in Chinese, Japanese, and American samples. Sociology of Religion, 54, 295-302.

McClenon, J. (1994). Wondrous events: Foundations of religious beliefs. Philadelphia, PA: University of Pennsylvania Press.

Moody, R. (1978). Life after life. Covington, GA: Mockingbird Books.

Moraldi, L. (1987). Nach dem Tode: Jenseitsvorstellungen von den Babyloniern bis zum Christentum. Köln: Benzinger.

Noyes, R., and Kletti, R. (1977). Panoramic memory: A response to the threat of death. Omega, 8, 181-194.

Osis, K., and Haraldsson, E. (1977). At the hour of death. New York, NY: Avon.

Ring, K. (1980). Life at death: A scientific investigation of the near-death experience. New York, NY: William Morrow.

Ring, K. (1982). Frequency and stages of the prototypic near-death experience. In C. R. Lundahl (Ed.), A collection of near-death research readings (pp. 110-159). Chicago, IL: Nelson-Hall.

Ring, K. (1992). The Omega Project: Near-death experiences, UFO encounters, and mind at large. New York, NY: William Morrow.

Roberts, G., and Owen, J. (1988). The near-death experience. British Journal of Psychiatry, 153, 607-617.

Sabom, M. (1982). Recollections of death: A medical investigation. New York, NY: Harper and Row.

Schröter-Kunhardt, M. (1990). Erfahrungen Sterbender während des klinischen Todes: Eine Brücke zwischen Medizin und Religion. Zeitschrift für Allgemeinmedizin 66, 1014-1021.

Schröter-Kunhardt, M. (1993). A review of near death experiences. Journal of Scientific Exploration 7, 219-239.

Scott, L. G. (1931). Dying as a liberation of consciousness: Record of a personal experience. Journal of the American Society for Psychical Research, 25, 113-117.

Sutherland, C. (1990). Changes in religious beliefs, attitudes, and practices following near-death experiences: An Australian study. Journal of Near-Death Studies, 9, 21-31.

Sutherland, C. (1995). Reborn in the light: Life after near-death experiences. New York, NY: Bantam. (Originally published in Australia in 1992 as Transformed by the light.)

Tucker, L. (1943). Clerical errors. New York, NY: Harper and Row.

Wells, A. D. (1993). Reincarnation beliefs among near-death experiencers. Journal of Near-Death Studies, 12, 17-34.

Zaleski, C. (1995). Otherworld journeys: Accounts of near-death experience from medieval and modern times. New York, NY: Oxford University Press. 